\title{
Entire Reflective Object Surface Structure Understanding
}

Qinglin Lu ${ }^{1}$

qinglin.lu@u-bourgogne.fr

Olivier Laligant ${ }^{1}$

http://le2i.cnrs.fr/-Olivier-Laligant- ?lang=fr

Eric Fauvet ${ }^{1}$

http://le2i.cnrs.fr/-Eric-Fauvet-?lang=fr

Anastasia Zakharova ${ }^{2}$

http://Imi.insa-rouen.fr/membres/10-membres/maitres-de-conference/29-zakharova-anastasia.html
${ }^{1}$ University of Burgundy

Le2i UMR 6306 CNRS

12,Rue de la Fonderie,71200,France

2 INSA Rouen LMI EA3226

Avenue de l'Université,76800,France
The presence of reflection on a surface has been a long-standing problem for object recognition since it brings negative effects on object's color, texture and structural information. Because of that, it is not a trivial task to recognize the surface structure affected by the reflection, especially when the object is entirely reflective. Most of the cases, reflection is considered as noise. In this paper, our proposed approach extracts reflection motion features in the image sequence as spatiotemporal information, then subsegment object by taking these features in order to understand the OSS. The setup of our method is straightforward, the positions of camera and object are fixed, however the light source is moving around the object in order to produce reflection particles (RP) on the object surface. While the RP are moving on the object surfaces, their positions, directions, and velocities are extracted in each frame as reflection motion features. These features are matched in all the frames for tracking RP in the whole sequence. We assume that the RP move smoothly along an elementary continuous surface and irregularly while passing from one surface to another. Thus, we break tracking when the motion features are irregularly compare to that in the previous frames. This guarantees to keep the trajectory of a moving RP stay on one elementary continuous surface. Later, one elementary continuous surface is segmented by employing flood fill method [5] which takes the positions in the trajectory as seeds. As this process iteratively covers all the trajectories, different surfaces of the object could be respectively labeled.

The RP are extracted by the motion history image $[1,3](M H I)$. The $M H I H_{\tau}(x, y, t)$ can be computed from an update function $\Psi_{\tau}(x, y, t)$ :

$$
H_{\tau}(x, y, t)=\left\{\begin{array}{lll}
\tau & \text { if } & \Psi_{\tau}(x, y, t)=1 \\
\max \left(0, H_{\tau}(x, y, t-1)-\delta\right) & \text { if } & \Psi_{\tau}(x, y, t)=0
\end{array}\right.
$$

Precisely, if $\Psi_{\tau}(x, y, t)=1$, then the pixel at position $(x, y)$ in $t$-th frame has moved. The duration $\tau$ decides the temporal extent of the movement, and $\delta$ is the decay parameter. This leads to a static scalar valued image where the more recently moving pixels are brighter.

The tracking of RP suffers from several problems: the high frequency of appearance and disappearance of the RP, the shape evolution of the $\mathrm{RP}$, as well as multiple reference RP need to be tracked in the same time. Our tracker is initialized for each detection of a reference $\operatorname{RP}\left(C_{i}^{t}\right)$. During tracking RP in frames, positions of all tracking results are saved as the moving trajectory. The trajectory of $C_{i}$ is denoted as $T\left(C_{i}\right)=$ $\left\{p_{i}^{1}, p_{i}^{2}, \ldots p_{i}^{t},\right\}$. One trajectory is considered as one label for a continuous surface on the object. As the RP could go through one surface in different directions, we save trajectories respectively for each direction. In this case, it ensures that one trajectory labels only one surface. On the other hand, some trajectories label the same surface.

Segmentation of the elementary continuous surfaces is to describe the surface structure of the object. As some trajectories are labeling the same surface, an iterative flood fill function is applied to merge the segmentation results of different trajectories on the same surface. The seeds which need to be flood filled are systematic sampled positions with a skip of 5 in the trajectory. Since the surface is elementary continuous, a trajectory can cover the surface regions with different brightness levels, the flood fill produces only one surface and the reflection don't produce additional sebsegments. The flood fill method which we used during the segmentation is the same for the reflection particle detection.

Since the trajectories do not have the same length, we put trajectories in an order by increasing lengths and systematic sample the positions by a skip of 5 , flood fill from sampled seeds in shorter trajectories to the sampled seeds in longer trajectories. In this case, the parts of one surface which has already been labeled by seeds in shorter trajectories could be merged into other parts of this surface by the labeling of seeds in longer trajectories. As the reflection on the surface is highly variable, the segmentation phase might not cover the whole surfaces. In consequence, the final processing fills the holes which are surrounded by segmented regions with the surrounding color.

The experiments are conducted in using the camera with the resolution of $5 \mathrm{Mpx}$. A LED grow light is used to produce reflections on the object. For the outdoor experiments, two projectors are used. The number of acquired frames is depending on the complexity of the object surfaces and the number of light sources. In order to keep the number of acquired image not expanded, our LED grow light contains 30 light spots. The ground-truth images are manually labeled according to the $3 \mathrm{D}$ models of the objects which are obtained by a non-contact 3D digitizer VI-910.

Our primary contributions are: (1)We introduce an effective subsegmentation method for the reflective surface structure understanding (on both specular and transparent surfaces). (2) Instead of removing reflection, we study the reflection motion and we consider it as additional information for sub-segmentation. (3) We use the reflection motion features as spatiotemporal coherence for video segmentation and fine-attributes for OSS understanding.

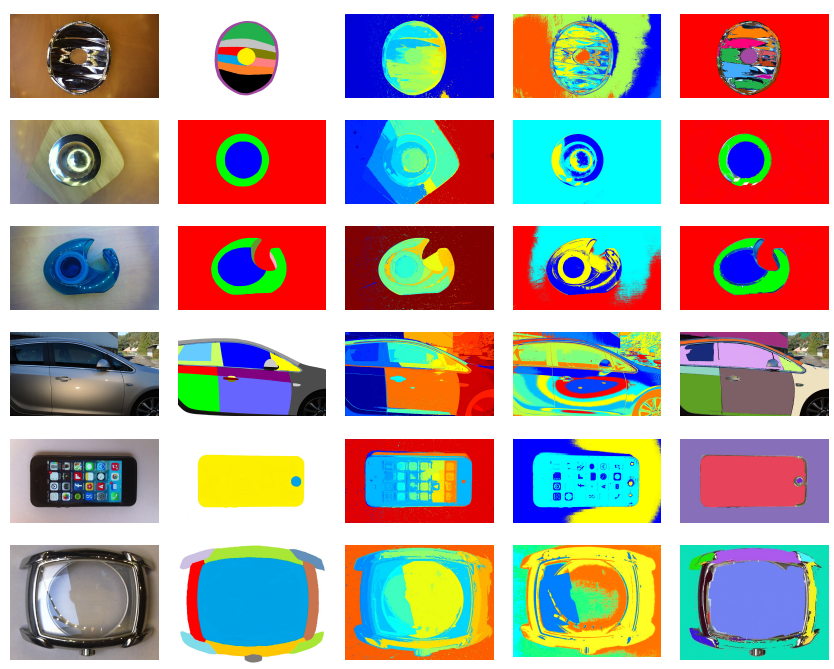

Figure 1: First column: original images. Second column: ground-truth segmentation. Third column: k nearest neighborhood graph-based segmentation [4]. Forth column: EM segmentation [2]. Last column: Segmentation by our proposed method. (better see in color)

[1] Md. Atiqur Rahman Ahad, J. K. Tan, H. Kim, and S. Ishikawa. Motion history image: Its variants and applications. Machine Vision and Applications, 23:255-281, 2012.

[2] C. Carson, S. Belongie, H. Greenspan, and J. Malik. Blobworld: Image segmentation using expectation-maximization and its application to image querying. IEEE Transactions on Pattern Analysis and Machine Intelligence, 24:1026-1038, 1999.

[3] J. Davis. Hierarchical motion history images for recognizing human motion. IEEE workshop DREV, 2001.

[4] P. Felzenszwalb and D. Huttenlocher. Efficient graph-based image segmentation. International Journal of Computer Vision, 59, 2004.

[5] A. Treuenfels. An efficient flood visit algorithm. C/C++ Users Journal, 12, 1994. 